\title{
Maximal steered coherence and its conversion to entanglement in multiple bosonic reservoirs
}

\author{
Xiao-Xiao $\mathrm{Xu}$ and Ming-Liang $\mathrm{Hu}^{\text {* }}$ \\ School of Science, Xi'an University of Posts and Telecommunications, Xi'an 710121, China
}

\begin{abstract}
The remote control of coherence is a crucial step for its application in quantum computation. We investigate the maximal steered coherence (MSC) and its conversion to entanglement in multiple bosonic reservoirs. It is shown that the MSC decays with time in the Markovian regime and behaves as damped oscillations in the nonMarkovian regime. The MSC can also be connected directly to the strength of non-Markovianity, through which we show that it can be noticeably enhanced by taking full advantage of the non-Markovian effects. Besides, the MSC can be converted completely to entanglement via optimal incoherent operation applied to the steered qubit and an incoherent ancilla which is immune of decoherence, and the generated entanglement is stronger than the shared entanglement in prior. These findings suggest a potential way for remotely generating and manipulating coherence and entanglement in noisy environments.
\end{abstract}

PACS numbers: 03.65.Yz, 03.67.Bg, 03.65.Ud, 03.65.Ta

\section{INTRODUCTION}

As the fundamental characteristics differentiating a quantum system from its classical counterpart, quantum coherence has remained one of the research focuses of the quantum community for over a century [1]. In the past few decades, quantum coherence further found its application in the emerging fields of quantum computation, quantum communication, and quantum metrology [2]. Recently, it is attracting growing interest once again, motivated by the formulation of the resource theory of coherence which sets the stage for analyzing quantitatively the decoherence mechanism of the open systems [3-5]. Within this framework, a number of coherence measures have also been introduced [5-11], starting from which researchers further studied explicitly its role in the tasks of state merging [12], deterministic quantum computation with one qubit [13], phase discrimination [9, 14], subchannel discrimination [10], and the Deutsch-Jozsa algorithm [15].

Although quantum coherence is defined for a single-partite system, it is intimately related to different quantum correlations. Their interrelations could be revealed by dividing a composite system into different subsystems and then analyzing distribution of the coherence among these subsystems [6, 11, 16-18]. From a practical point of view, it is also meaningful to explore their interrelations from an operational perspective, e.g., by considering the steered coherence of a bipartite system via local operations and classical communication (LOCC) [19-23]. Actually, the coherence distillation and dilution tasks have been extensively studied based on different quantum operations [24-29]. For a bipartite system $A B$, it has also been shown that one cannot create coherence on $B$ via LOCC only when $\rho_{A B}$ is a quantum-incoherent state [30]. For the steered coherence averaged over all the mutually unbiased bases, it has been shown that it may capture a kind of quantum correlation stronger than entanglement [21, 22]. Moreover, the maximal steered coherence (MSC) can be linked to discord-like correlations, e.g., it vanishes for the zero-discord

*Electronic address: mingliang0301@163.com states [19]. Here, by saying the MSC, we mean the steered coherence on $B$ maximized over the positive-operator-valued measure (POVM) on $A$.

Quantum coherence of a system $S$ could be converted to entanglement via incoherent operations on $S$ and an ancilla [6]. Moreover, the amount of coherence in a state can be enhanced by performing a unitary operation on it $[31-33]$. Thus for a bipartite system $A B$ shared between two participants, one might realize the control of coherence on $B$ via LOCC and the controlled creation of entanglement between $B$ and an incoherent ancilla $C$ by utilizing the MSC (or the MSC further maximized over the unitary transformations) on $B$. Besides, the ancillary system can be initialized in a state that is immune of decoherence in general, so it may be able to suppress the detrimental effect of decoherence on entanglement.

In this paper, we investigate the MSC of two qubits $A$ and $B$ immersed in two groups of multiple bosonic reservoirs, aimed at revealing the (non-)Markovian effects triggered by different physical mechanisms on its behaviors and its conversion to entanglement. We will show that the MSC decays exponentially with time in the Markovian regime and behaves as damped oscillations of time in the non-Markovian regime. Besides, the entanglement converted from the MSC is stronger than the entanglement of $A B$. This observation manifests efficiency of the active quantum operations on remote control of coherence and entanglement in noisy environments.

This paper is arranged as follows. In Section II we recall measures of the MSC, then in Section III we give solution of the multiple reservoirs and the non-Markovianity. In Sections IV and $\mathrm{V}$, we present an analysis of the decay behaviors of the MSC and a comparison of the entanglement converted from the MSC with the shared entanglement of two participants. Finally, Section $\mathrm{VI}$ is devoted to a summary of the main results.

\section{MAXIMAL STEERED COHERENCE}

We consider the steering of coherence between two participants Alice $(A)$ and $\operatorname{Bob}(B)$ who share a two-qubit state $\rho_{A B}$ in prior. First, Alice performs the POVM measurements $M$ on 


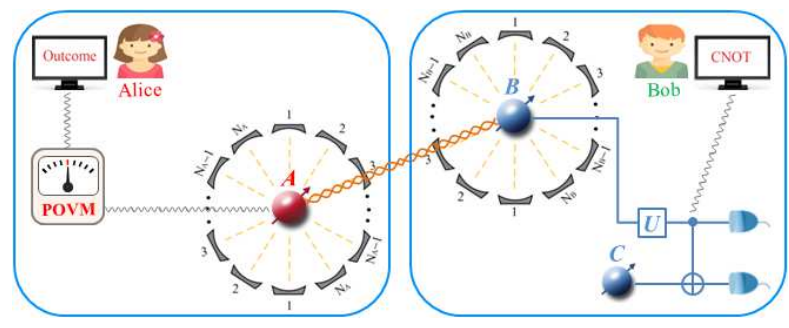

FIG. 1: Schematic picture for steering Bob's coherence via LOCC in multiple bosonic reservoirs (which could be realized, e.g., by two sets of lossy cavities) and conversion of the MSC to entanglement of $B C$ under the сNOT operation. Here, the control qubit $B$ is entangled with qubit $A$, while the target qubit $C$ is initialized in the ground state $|0\rangle$ which is immune of the bosonic reservoirs.

qubit $A$, after which the state of qubit $B$ collapses to

$$
\rho_{B \mid M}=\operatorname{tr}_{A}\left(M \otimes \mathbb{1} \rho_{A B}\right) / p_{M},
$$

where $p_{M}=\operatorname{tr}\left(M \otimes \mathbb{1} \rho_{A B}\right)$ is the probability for attaining $\rho_{B \mid M}$. To quantify the net amount of coherence generated on $B$, Bob chooses the reference basis $\left\{\left|\xi_{i}\right\rangle\right\}$ spanned by the eigenbasis of $\rho_{B}=\operatorname{tr}_{A} \rho_{A B}$ in which there is no initial coherence on $B$. Hence, the MSC attainable by Bob will be given by [19]

$$
C_{\mu}^{\mathrm{msc}}\left(\rho_{A B}\right)=\inf _{\left\{\left|\xi_{i}\right\rangle\right\}}\left\{\max _{M \in \mathrm{POVM}} C_{\mu}^{\left\{\left|\xi_{i}\right\rangle\right\}}\left(\rho_{B \mid M}\right)\right\},
$$

where the infimum over $\left\{\left|\xi_{i}\right\rangle\right\}$ is introduced as $\rho_{B}$ may be degenerate, while $C_{\mu}^{\left\{\left|\xi_{i}\right\rangle\right\}}\left(\rho_{B \mid M}\right)$ represents a measure of coherence in $\rho_{B \mid M}$. In this paper, we will consider two well-accepted measures of coherence, that is, the $l_{1}$ norm of coherence and the relative entropy of coherence given by [5]

$$
\begin{aligned}
& C_{l_{1}}^{\left\{\left|\xi_{i}\right\rangle\right\}}\left(\rho_{B \mid M}\right)=\sum_{i \neq j}\left|\left\langle\xi_{i}\left|\rho_{B \mid M}\right| \xi_{j}\right\rangle\right|, \\
& C_{\mathrm{re}}^{\left\{\left|\xi_{i}\right\rangle\right\}}\left(\rho_{B \mid M}\right)=S\left[\left(\rho_{B \mid M}\right)_{\text {diag }}\right]-S\left(\rho_{B \mid M}\right),
\end{aligned}
$$

where $\left(\rho_{B \mid M}\right)_{\text {diag }}$ denotes the state obtained by deleting all the off-diagonal elements of $\rho_{B \mid M}$, and $S(\cdot)$ is the von Neumann entropy of the corresponding state. Of course, the MSC can also be quantified by other coherence measures, but their behaviors are qualitatively the same, thus we will do not consider them one by one.

\section{SOLUTION OF THE MODEL}

We consider a central system consists of two noninteracting two-level atoms (serve as the qubits) labeled as $A$ and $B$. When they are coupled independently to two sets of multiple bosonic reservoirs as shown in Fig. 1 the total Hamiltonian is given by $\hat{H}=\hat{H}_{A}+\hat{H}_{B}$, where the single "qubit+reservoir" Hamiltonian $\hat{H}_{S}(S=A$ or $B)$ reads (in units of $\hbar$ )

$$
\hat{H}_{S}=\frac{1}{2} \omega_{0} \sigma_{z}+\sum_{n=1}^{N_{S}} \sum_{k}\left[\omega_{n, k} b_{n, k}^{\dagger} b_{n, k}+g_{n, k}\left(b_{n, k} \sigma_{+}+\text {H.c. }\right)\right],
$$

where $\omega_{0}$ is the transition frequency between the ground state $|0\rangle$ and the excited state $|1\rangle$ of the two qubits, $\sigma_{ \pm}=\left(\sigma_{x} \pm\right.$ $\left.i \sigma_{y}\right) / 2$ are the raising and lowering operators, $\sigma_{x, y, z}$ are the three Pauli operators, and $b_{n, k}\left(b_{n, k}^{\dagger}\right)$ is the annihilation (creation) operator of the $n$th reservoir's field mode $k$ with frequency $\omega_{n, k}$, while its coupling strength to the qubit is $g_{n, k}$. Moreover, $N_{S}$ is the number of reservoirs acting on $S$, which may be implemented by $N_{S}$ pairs of lossy cavity mirrors 34 36] as sketched in Fig. [1.

For the qubit $S$ ( $S=A$ or $B$ ) being prepared initially in the state $\rho_{S}(0)$ and there is no initial correlation between $S$ and the reservoirs, its evolved state after tracing over the $N_{S}$ reservoirs can be obtained as [35, 36]

$$
\rho_{S}(t)=\left(\begin{array}{cc}
\rho_{S}^{11}(0)\left|p_{S}(t)\right|^{2} & \rho_{S}^{10}(0) p_{S}(t) \\
\rho_{S}^{01}(0) p_{S}^{*}(t) & 1-\rho_{S}^{11}(0)\left|p_{S}(t)\right|^{2}
\end{array}\right),
$$

where $\rho_{S}^{i j}(0)=\left\langle i\left|\rho_{S}(0)\right| j\right\rangle$, and $p_{S}(t)$ is a time-dependent parameter determined by the spectra of these reservoirs. We will consider the reservoirs with the Lorentzian spectrum $J_{n}(\omega)$ for which $p_{S}(t)$ is analytically solvable. Here, $J_{n}(\omega)=$ $\gamma_{n} \lambda_{n}^{2} /\left\{\left[2 \pi\left[\left(\omega-\omega_{0}\right)^{2}+\lambda_{n}^{2}\right]\right\}\right.$, where $\lambda_{n}$ denotes the spectral width of the $n$th reservoir whose reciprocal determines its characteristic correlation time, while the reciprocal of $\gamma_{n}$ determines the relaxation time of the qubit [37]. In the following, we focus on the case that all the reservoirs are the same (i.e., $\lambda_{n} \equiv \lambda$ and $\left.\gamma_{n} \equiv \gamma, \forall n\right)$, then in the non-Markovian regime, one has

$$
p_{S}(t)=e^{-\frac{1}{2} \lambda t}\left(\cos \frac{d_{S} t}{2}+\frac{\lambda}{d_{S}} \sin \frac{d_{S} t}{2}\right)
$$

where $d_{S}=\left(\left|\lambda^{2}-2 N_{S} \gamma \lambda\right|\right)^{1 / 2}$. In the Markovian regime, $p_{S}(t)$ has a similar form but with $\cos (\cdot)$ and $\sin (\cdot)$ being replaced by $\cosh (\cdot)$ and $\sinh (\cdot)$, respectively. Here, the (non)Markovianity can be distinguished by the relative magnitudes of $\lambda$ and $\gamma$. For $\lambda>2 N_{S} \gamma, p_{S}(t)$ decays exponentially with time and the evolution is Markovian. For $\lambda<2 N_{S} \gamma$, $p_{S}(t)$ oscillates with time which is an embodiment of the nonMarkovian dynamics.

For given $\lambda$ and $\gamma$, one can see from Eq. (6) that there exists a critical value for the number $N_{S}$ of reservoirs after which the non-Markovianity occurs. Such a critical value can be obtained as $N_{S, \text { cr }}=\lfloor\lambda / 2 \gamma\rfloor+1(\lfloor x\rfloor$ is the nearest integer not larger than $x$ ). The non-Markovianity occurs when $N_{S} \geqslant N_{S \text {,cr }}$ can be confirmed via the BreuerLaine-Piilo measure of non-Markovianity defined as $\mathcal{N}_{\text {BLP }}=$ $\max _{\rho_{1,2}(0)} \int_{\varepsilon>0} \varepsilon\left[t, \rho_{1,2}(0)\right] \mathrm{d} t[38]$, where $\varepsilon\left[t, \rho_{1,2}(0)\right]$ is the time derivative of the trace distance between $\rho_{1}(t)$ and $\rho_{2}(t)$ defined by $D\left[\rho_{1}(t), \rho_{2}(t)\right]=\operatorname{tr}\left|\rho_{1}(t)-\rho_{2}(t)\right| / 2$. As has been shown in Ref. [36], for the optimal $\rho_{1,2}^{\mathrm{op}}(0)$ (i.e., the eigenstates of $\left.\sigma_{x}\right)$, one has $D\left[\rho_{1}(t), \rho_{2}(t)\right]=\left|p_{S}(t)\right|$, which vanishes at the critical times $t_{z, l}=2\left(l \pi-\vartheta_{S}\right) / d_{S}$ and reaches to its peak at $t_{p, l}=2(l-1) \pi / d_{S}$, with $\vartheta_{S}=\arctan \left(d_{S} / \lambda\right)$ and $l \in \mathbb{N}$. From these results one can obtain $\mathcal{N}_{\mathrm{BLP}}=\sum_{l=1}^{\infty} e^{-l \lambda \pi / d_{S}}$. It increases with the increase of $N_{S}$ when $N_{S} \geqslant N_{S \text {,cr. }}$. Besides, one can also quantify the non-Markovianity based on the backflow ra- 
tio of information, which we define it as

$$
\mathcal{N}_{\mathrm{BRI}}=\frac{\int_{\varepsilon>0} \varepsilon\left[t, \rho_{1,2}^{\mathrm{op}}(0)\right] \mathrm{d} t}{\int_{\varepsilon<0} \varepsilon\left[t, \rho_{1,2}^{\mathrm{op}}(0)\right] \mathrm{d} t},
$$

which is the ratio of the time-integration of $\varepsilon$ over all the time intervals $\left[t_{z, l}, t_{p, l+1}\right]$ in which $\varepsilon$ is positive and that over all the time intervals $\left[t_{p, l}, t_{z, l}\right]$ in which $\varepsilon$ is negative. Here, one may interpret the term on the numerator (denominator) of Eq. (7) as the backflow (outflow) of information. Different from $\mathcal{N}_{\text {BLP }}$ which contains a summation over all $l \in \mathbb{N}$, one can show that in every time interval $\left[t_{p, l}, t_{p, l+1}\right](l \in \mathbb{N})$, the ratio of the timeintegration of the two terms in Eq. (7) is completely the same. Therefore, one has the following exact result:

$$
\mathcal{N}_{\mathrm{BRI}}=e^{-\lambda \pi / d_{S}},
$$

which is also larger than zero when $N_{S} \geqslant N_{S \text {,cr. }}$ Besides, it also increases with an increase in $N_{S}$ when $N_{S} \geqslant N_{S, \mathrm{cr}}$.

\section{MSC IN MULTIPLE BOSONIC RESERVOIRS}

While the authors in Ref. [36] considered the issue of a single qubit transversally coupled to the multiple bosonic reservoirs, it is also meaningful to generalize this theoretical model to the two-qubit case for which one can further investigate effects of the multiple bosonic reservoirs on controlling quantum correlations. As a matter of fact, for two qubits coupled independently to two bosonic reservoirs with $N_{A}=N_{B}=1$, the dynamical behaviors of entanglement [35, 39-41], discord-like correlations [42-44], and entropic uncertainty relation [45], have already been investigated, and it is found that the non-Markovian effect triggered by increasing the coupling strength is beneficial to them. Then it is natural to ask whether the non-Markovian effect triggered by increasing the number of reservoirs acting on each qubit is beneficial for protecting coherence and entanglement of a state.

In this section, we explore behaviors of MSC for two qubits coupled independently to two groups of multiple reservoirs as shown in Fig. 11. We will consider two slightly different cases: the case of $N_{A}=N_{B}$ for which we call it symmetric reservoirs and the case of $N_{A} \neq N_{B}$ for which we call it asymmetric reservoirs. We consider the initial Bell-like state $|\Psi\rangle=\alpha|10\rangle+\beta|01\rangle\left(|\alpha|^{2}+|\beta|^{2}=1\right)$. Note that although the two qubits might be initially quantum correlated depending on the parameters $\alpha$ and $\beta$, there is no direct interaction neither between the two qubits nor between the two multiple bosonic reservoirs. Hence, one can derive the evolving state of the two qubits based on the method given in Ref. [39]. To be explicit, we write the elements of $\rho_{S}(t)$ of Eq. (5) as $\rho_{S}^{i i^{\prime}}(t)=\sum_{l l^{\prime}} S_{i i^{\prime}}^{l l^{\prime}}(t) \rho_{S}^{l l^{\prime}}(0)\left(i, i^{\prime}, l, l^{\prime} \in\{0,1\}\right)$, from which one can obtain the nonzero $S_{i i^{\prime}}^{l l^{\prime}}(t)$ as

$$
\begin{aligned}
& S_{11}^{11}(t)=\left|p_{S}\right|^{2}, S_{10}^{10}(t)=p_{S}, S_{01}^{01}(t)=p_{S}^{*}, \\
& S_{00}^{00}(t)=1, S_{00}^{11}(t)=1-\left|p_{S}\right|^{2},
\end{aligned}
$$

where $S=A$ or $B$. By substituting these into Eq. (5) of Ref.
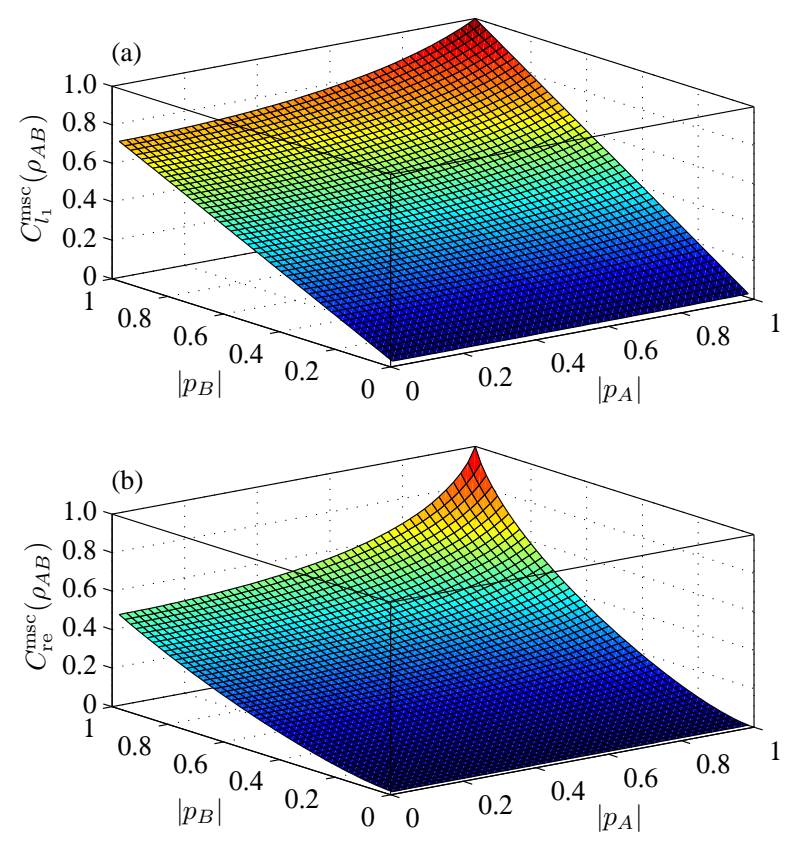

FIG. 2: The $\operatorname{MSC} C_{\mu}^{\text {msc }}\left(\rho_{A B}\right)\left(\mu=l_{1}\right.$ or re) versus $\left|p_{A}\right|$ and $\left|p_{B}\right|$ for the initial state $\left|\Psi^{+}\right\rangle$of qubits $A$ and $B$.

[39] and using the fact that $p_{S} \in \mathbb{R}$, one can obtain

$$
\rho_{A B}(t)=\left(\begin{array}{cccc}
0 & 0 & 0 & 0 \\
0 & \left|\alpha p_{A}\right|^{2} & \alpha \beta^{*} p_{A} p_{B} & 0 \\
0 & \alpha^{*} \beta p_{A} p_{B} & \left|\beta p_{B}\right|^{2} & 0 \\
0 & 0 & 0 & 1-\left|\alpha p_{A}\right|^{2}-\left|\beta p_{B}\right|^{2}
\end{array}\right),
$$

and we will focus on the case of $\alpha \beta \neq 0$ unless specifically stated based on the consideration that Alice cannot steer Bob's coherence at all when $\alpha \beta=0$.

For the two-qubit states, as has been explained in Ref. [19], one only needs to take the maximization over the set of projective measurements $M=(\mathbb{1}+\vec{m} \cdot \sigma) / 2$, where $\sigma=\left(\sigma_{x}, \sigma_{y}, \sigma_{z}\right)$ and $\vec{m}=(\sin \theta \cos \phi$, $\sin \theta \sin \phi, \cos \theta)$, with $\theta$ and $\phi$ being the polar and azimuth angles, respectively. Then the postmeasurement state of qubit $B$ can be obtained as

$$
\rho_{B \mid M}=\left(\begin{array}{cc}
\frac{\left|\beta p_{B}\right|^{2} \sin ^{2}(\theta / 2)}{p_{M}} & \frac{e^{-i \phi} \alpha^{*} \beta p_{A} p_{B} \sin \theta}{2 p_{M}} \\
\frac{e^{i \phi} \alpha \beta^{*} p_{A} p_{B} \sin \theta}{2 p_{M}} & 1-\frac{\left|\beta p_{B}\right|^{2} \sin ^{2}(\theta / 2)}{p_{M}}
\end{array}\right),
$$

where $p_{M}=\left[1+\left(2\left|\alpha p_{A}\right|^{2}-1\right) \cos \theta\right] / 2$, so the $l_{1}$ norm of MSC remains zero for $\alpha \beta=0$, irrespective of $\theta$ and $\phi$. Otherwise, the optimal polar angle is $\theta_{0}=\arccos \left(1-2\left|\alpha p_{A}\right|^{2}\right)$, while the azimuth angle can take any value. As a result, one has

$$
C_{l_{1}}^{\mathrm{msc}}\left(\rho_{A B}\right)= \begin{cases}\frac{\left|\beta p_{B}\right|}{\sqrt{1-\left|\alpha p_{A}\right|^{2}}} & \text { if } \alpha \beta \neq 0, \\ 0 & \text { if } \alpha \beta=0,\end{cases}
$$

from which one can note that it is monotonic increasing functions of both $\left|p_{A}\right|$ and $\left|p_{B}\right|$. As has been shown in Section 

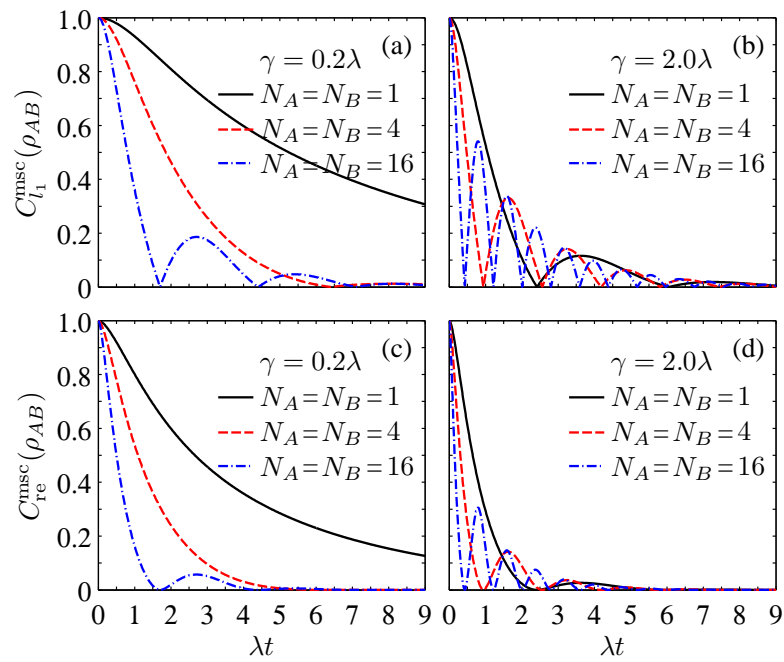

FIG. 3: The MSC $C_{\mu}^{\mathrm{msc}}\left(\rho_{A B}\right)\left(\mu=l_{1}\right.$ or re) versus $\lambda t$ for the initial state $\left|\Psi^{+}\right\rangle$with different $\gamma, N_{A}$, and $N_{B}$ in the symmetric Lorentzian reservoirs.

the decrease of $\left|p_{S}\right|(S=A$ or $B$ ) signifies an outflow of information to the reservoirs, while the increase of $\left|p_{S}\right|$ signifies a backflow of information to the system. Hence, for the initial state $|\Psi\rangle$ with $\alpha \beta \neq 0$, the backflow of information to either one of the multiple reservoirs is always beneficial for protecting the $l_{1}$ norm of MSC. But the decay rates with respect to $\left|p_{A}\right|$ and $\left|p_{B}\right|$ are somewhat different. More specifically, for the symmetric multiple reservoirs, one can obtain from Eq. (12) that the decay rate of the $l_{1}$ norm of MSC with respect to $\left|p_{A}\right|\left(p_{B}=p_{A}\right)$ increases with the increase of $\left|p_{A}\right|$. For the asymmetric multiple reservoirs, it decays linearly with the decrease of $\left|p_{B}\right|$ and the decay rate is independent of $\left|p_{B}\right|$, while for $p_{B} \neq 0$, it shows a parabolic decrease with the decrease of $\left|p_{A}\right|$, but the decay rate is not a constant function of $\left|p_{A}\right|$, see Fig. 22(a).

As for the relative entropy of MSC, it is also independent of the azimuth angle $\phi$, but it is difficult to obtain analytically the optimal polar angle $\theta_{0}$. So we have to optimize it numerically. Here, $C_{\mathrm{re}}^{\mathrm{msc}}\left(\rho_{A B}\right)$ can be written as

$$
C_{\mathrm{re}}^{\mathrm{msc}}\left(\rho_{A B}\right)=\max _{\{\theta\}}\left\{S\left[\left(\rho_{B \mid M}\right)_{\mathrm{diag}}\right]-S\left(\rho_{B \mid M}\right)\right\},
$$

and the corresponding numerical result is shown in Fig. 2 (b), from which one can see that its dependence on $\left|p_{A}\right|$ and $\left|p_{B}\right|$ is similar to that of the $l_{1}$ norm of MSC. The only difference is that $C_{\mathrm{re}}^{\mathrm{msc}}\left(\rho_{A B}\right)$ does not decrease linearly with the decreasing value of $\left|p_{B}\right|$.

In the following, we investigate the time dependence of the MSC. First, we consider the case of the symmetric Lorentzian reservoirs. For the initial state $|\Psi\rangle$ with $\alpha=\beta=1 / \sqrt{2}$ (we denote by it $\left|\Psi^{+}\right\rangle$for conciseness of later presentation), we show in Fig. 3 $C_{\mu}^{\text {msc }}\left(\rho_{A B}\right)\left(\mu=l_{1}\right.$ or re) versus $\lambda t$ with different $\gamma$. For the given parameters in this figure, $N_{S \text {,cr }}=3$ for $\gamma=0.2 \lambda$ and $N_{S, \text { cr }}=1$ for $\gamma=2.0 \lambda$. As expected, the MSC decreases monotonically with the evolving time in the Markovian regime, see the solid lines in Fig. [3 a) and (c). When
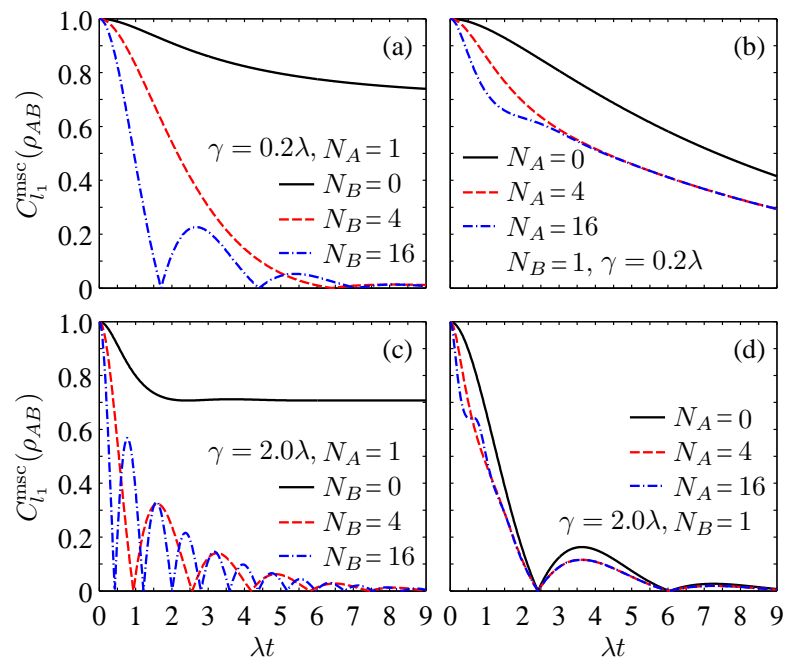

FIG. 4: The MSC $C_{l_{1}}^{\mathrm{msc}}\left(\rho_{A B}\right)$ versus $\lambda t$ for the initial state $\left|\Psi^{+}\right\rangle$with different $\gamma, N_{A}$, and $N_{B}$ in the asymmetric Lorentzian reservoirs.

the non-Markovian effects are triggered, either by increasing the coupling strength $\gamma$ or by increasing the number of reservoirs, both the $l_{1}$ norm and relative entropy of MSCs behave as damped oscillations (with the period $T=2 \pi / d_{S}$ ) with the time $t$ evolves and undergo sudden death at the critical times $t_{z, l}(l \in \mathbb{N})$. Physically, the decay behaviors are caused by the exponential term in Eq. (6), while the oscillations are due to the sine and cosine terms. Moreover, at the critical times $t_{p, l}$ $(l \in \mathbb{N})$, one has

$$
\left.C_{l_{1}}^{\mathrm{msc}}\left(\rho_{A B}\right)\right|_{t=t_{p, l}}= \begin{cases}\frac{|\beta| \mathcal{N}_{\mathrm{BRI}}^{l-1}}{\sqrt{1-|\alpha|^{2} \mathcal{N}_{\mathrm{BRI}}^{2(l-1)}}} & \text { if } \alpha \beta \neq 0, \\ 0 & \text { if } \alpha \beta=0,\end{cases}
$$

which shows that the peak values of the MSC in the nonMarkovian regime can always be enhanced by increasing the non-Markovianity of the multiple reservoirs.

Next, we consider the time dependence of the MSC in the asymmetric Lorentzian reservoirs. In Fig. 4 we show $C_{l_{1}}^{\text {msc }}\left(\rho_{A B}\right)$ versus $\lambda t$ for $\left|\Psi^{+}\right\rangle$with different $\gamma$. When both $N_{A}$ and $N_{B}$ are smaller than $N_{S \text {,cr }},\left|p_{S}(t)\right|$ shows a Markovian exponential decay, hence as expected, the MSC also decays exponentially with the time evolves, see the solid black lines displayed in Fig. 4. When one set of the multiple reservoirs is in the non-Markovian regime, it can be found from Fig. 4 (a) and (b) that for $N_{A}<N_{B}$, there are still revives of the MSC, while for $N_{A}>N_{B}$ the MSC decays monotonically. Such a difference is rooted in the asymmetric property of the MSC and could be explained from Eq. (12), as the enhancement of $\left|p_{A}\right|$ may do not suffice to compensate the loss of $\left|p_{B}\right|$ for the system parameters in Fig. 4 (b). Of course, one may observe a very weak revival of the MSC by increasing slightly $N_{B}$ (e.g., $N_{B}=2$ ). When the two reservoirs acting on the qubits $A$ and $B$ are already in the non-Markovian regime for $N_{A, B}=1$, as can be found from Fig. 4(c) and (d), the MSC also behaves as damped oscillations with the time evolves. But now the period 
and the peak values of the oscillations will be determined by both $p_{A}$ and $p_{B}$.

For the relative entropy of MSC, its behaviors in the asymmetric Lorentzian reservoirs are structurally similar to that of the $l_{1}$ norm of MSC, so we do not present the corresponding plots here.

As coherence itself is a precious resource for quantum computing and the related quantum tasks, it is vital to seek flexible methods to protect coherence. The previous studies showed that the decoherence effects could be suppressed by taking full advantage of the initial system-bath correlation [46], the correlations between consecutive actions of a noisy channel [47, 48], and the quantum-jump-based feedback [49]. Besides, the coherence might also be frozen under specific conditions [50-52]. Different from these methods for local controlling coherence, our results presented above suggest a flexible way for remotely controlling coherence in the noisy environments. In particular, such a steered coherence may be stronger than that of the single-qubit coherence under the same reservoirs. For example, if qubit $B$ is uncorrelated with qubit $A$ (so Alice cannot steer its coherence) and is initialized in the state $\alpha|1\rangle+\beta|0\rangle$, the $l_{1}$ norm of coherence for the time-evolved state of $B$ will be given by $C_{l_{1}}\left[\rho_{B}(t)\right]=2\left|\alpha \beta^{*} p_{B}\right|$. It is always weaker than the MSC of Eq. (12) when qubit $A$ is isolated from the reservoirs (i.e. $\left.p_{A}=1\right)$. Even when qubit $A$ is immersed in the bosonic reservoirs, $C_{l_{1}}\left[\rho_{B}(t)\right]$ is still weaker than the MSC in the parameter region of $|\alpha|^{2}<\left[1-\left(1-p_{A}^{2}\right)^{1 / 2}\right] / 2 p_{A}^{2}$.

\section{CONVERTING MSC TO ENTANGLEMENT}

Quantum coherence and entanglement are expensive resources for quantum communication such as quantum cryptography [53], motivated by which great efforts have been devoted to generating long-lived coherence and entanglement in various physical systems, e.g., the optomechanical system [54], ultracold atomic ensembles [55, 56], and cold ion [57]. Moreover, the coherence measures defined within the resource theoretic framework could be measured experimentally with elaborately designed techniques [58, 59] and are intimately related to quantum entanglement [3, 4, 60]. In particular, as was shown in Ref. [6], by performing the cNOT operation $\Lambda_{\mathrm{cNOT}}$ on the qubit $B$ which is coherent and an ancillary qubit $C$ initialized in the incoherent state $|0\rangle$, with $B(C)$ being the control (target) qubit, these two qubits will become entangled. If one uses concurrence [61, 62] as a measure of entanglement, then $C\left(\Lambda_{\mathrm{cNOT}}\left[\rho_{B} \otimes|0\rangle\langle 0|\right]\right)=C_{l_{1}}\left(\rho_{B}\right)[6]$, i.e., all the coherence in $\rho_{B}$ is converted to entanglement of $B C$ via the incoherent operation $\Lambda_{\mathrm{CNOT}}$.

The above finding shows a way to control the entanglement of Bob's qubits. For the scenario we considered in Fig. 1, the ancillary qubit $C$ is initialized in the ground state $|0\rangle$ and will be immune of the reservoirs. Hence, the generated entanglement in $B C$ equals to the $l_{1}$ norm of coherence in $\rho_{B \mid M}$. For the optimal state $\rho_{B \mid M \text {,op }}$ (i.e., the state $\rho_{B \mid M}$ with $\theta=\theta_{0}$ ), one has

$$
C\left(\Lambda_{\mathrm{cNoT}}\left[\rho_{B \mid M, \mathrm{op}} \otimes|0\rangle\langle 0|\right]\right)=C_{l_{1}}^{\mathrm{msc}}\left(\rho_{A B}\right),
$$

that is, the amount of created entanglement in $B C$ (measured by concurrence) equals exactly to the $l_{1}$ norm of MSC for $\rho_{A B}$. This gives an operational interpretation to the MSC.

From Eq. (10) one can obtain the concurrence of $\rho_{A B}$ as $C\left(\rho_{A B}\right)=2\left|\alpha \beta^{*} p_{A} p_{B}\right|$. As a result,

$$
\frac{C\left(\rho_{A B}\right)}{C\left(\Lambda_{\mathrm{cNoT}}\left[\rho_{B \mid M, \mathrm{op}} \otimes|0\rangle\langle 0|\right]\right)}=2\left|\alpha p_{A}\right| \sqrt{1-\left|\alpha p_{A}\right|^{2}} \leqslant 1,
$$

thus the generated entanglement in $B C$ is always stronger than the entanglement of $\rho_{A B}(t)$ whenever the reservoirs are present (i.e., $\left|p_{A, B}\right|<1$ ). Even for the ideal case (i.e., $p_{A, B}=1$ ), the equality holds only when $\rho_{A B}(t)$ belongs to one of the Bell states. In Ref. [63], a scheme for recovering entanglement via local operations is proposed. Our scheme is different from that in Ref. [63] as it suggests an efficient way for remotely instead of locally manipulating entanglement in noisy environments.

One might also concern the amount of generated entanglement in $B C$ without Alice's steering. But for such a situation, one has $C\left(\Lambda_{\mathrm{cNoT}}\left[\rho_{B}(t) \otimes|0\rangle\langle 0|\right]\right)=0$ for $\rho_{A B}(t)$ of Eq. 10). This consolidates the fact that with the help of the prior shared entanglement, Alice can control efficiently the entanglement of Bob's qubits.

Of course, the enhancement of the generated entanglement in $B C$ compared to $C\left(\rho_{A B}\right)$ is at the expense of reducing the success probability. In fact, the optimal success probability $p_{M, \text { op }}$ (i.e., the probability of attaining $\rho_{B \mid M, \text { op }}$ ) is given by

$$
p_{M, \text { op }}=2\left|\alpha p_{A}\right|^{2}\left(1-\left|\alpha p_{A}\right|^{2}\right),
$$

then one can see that the probability cannot exceed $50 \%$. To maximize the generated entanglement in $B C$, one can choose the initial state $\left|\Psi^{+}\right\rangle$for which $\left|\alpha p_{A}\right|^{2} \leqslant 1 / 2$. As a consequence, $p_{M, \text { op }}$ decreases with the decrease of $\left|p_{A}\right|$. For the case of qubit $A$ being isolated perfectly from the reservoirs, one has $p_{M, \mathrm{op}}=50 \%$.

Note that the coherence of a state could be enhanced by performing a unitary operation $U$ on it. For the single-qubit state $\rho$, the maximal coherence under unitary operations is given by $C_{l_{1}, \mathrm{op}}(\rho)=|\boldsymbol{x}|$, where $\boldsymbol{x}=\left(x_{1}, x_{2}, x_{3}\right)$, with $x_{i}=\operatorname{tr}\left(\rho \sigma_{i}\right)$ and the optimal $U_{\text {op }}$ can be constructed by the eigenbasis of $\rho$ [31, 32]. For $\rho_{B \mid M}$ of Eq. (11), if Bob applies $U_{\text {op }}$ to the qubit $B$ after Alice's optimal measurements (i.e., $\theta=\theta_{0}$ ), the optimized $l_{1}$ norm of MSC on $B$ will be given by

$$
C_{l_{1}, \mathrm{op}}^{\mathrm{msc}}\left(\rho_{B \mid M}\right)=\sqrt{1-\frac{\left|\beta p_{B}\right|^{2}\left(1-\left|\alpha p_{A}\right|^{2}-\left|\beta p_{B}\right|^{2}\right)}{\left(1-\left|\alpha p_{A}\right|^{2}\right)^{2}}} .
$$

Such an optimized $l_{1}$ norm of MSC is stronger than that of Eq. (12), hence can be used to create stronger entanglement in $B C$ than that given in Eq. 15). One can also note from the above equation that $C_{l_{1}, \mathrm{op}}^{\mathrm{msc}}\left(\rho_{B \mid M}\right)=1$ when $\beta=0$. This is because for such a special case, $\rho_{B}(t) \equiv|0\rangle\langle 0|$ and $U_{\text {op }}$ transforms it to the maximally coherent state [3]. But this lost the original idea of steering as Alice cannot steer Bob's coherence if $\alpha \beta=0$.

Finally, we would like to mention that when considering the initial state $|\Phi\rangle=\alpha|11\rangle+\beta|00\rangle, \rho_{B \mid M}$ can be obtained in a similar way, from which one can obtain that the $l_{1}$ norm of MSC is completely the same to that for the initial state $|\Psi\rangle$. For the 
relative entropy of MSC, although their strengths are somewhat different for the initial states $|\Phi\rangle$ and $|\Psi\rangle$, their behaviors are qualitatively the same. As a consequence, the findings we presented above also apply to the case of two qubits initialized in the Bell-like state $|\Phi\rangle$.

\section{SUMMARY AND DISCUSSION}

To summarize, we have investigated the control of the MSC for two qubits coupled independently to two groups of multiple bosonic reservoirs. For the two qubits being initialized in the Bell-like states, we showed that when the number of reservoirs acting on each qubit is smaller than a critical value, the MSC shows an exponential decay with time. But when it exceeds this critical value, the MSC turns to behave as damped oscillations, where the peak values of these oscillations can always be enhanced by increasing the non-Markovianity of the reservoirs. Besides, apart from the case of qubit $B$ being isolated perfectly from the reservoirs, the MSC will decay to zero in the infinite-time limit. Moreover, we have further shown that by performing the CNOT operation to the qubit $B$ and an ancillary qubit $C$ initialized in the ground state which is immune of the reservoirs, the MSC will be completely converted to entanglement in $B C$, and such an entanglement generated with the help of LOCC is stronger than or equals to the entanglement shared between Alice and Bob. We hope these observations may shed some light on revealing the interplay between the unavoidable decoherence of a system and efficiency of the active quantum operations on protecting coherence. From a practical point of view, it may also provide an alternate for remotely generating and manipulating coherence and entanglement in noisy environments.

Although we considered only the bosonic reservoirs with Lorentzian spectrum, the investigations in this work can be generalized immediately to other kinds of bosonic reservoirs, e.g., the sub-Ohmic, Ohmic, and super-Ohmic reservoirs [64], under which the postmeasurement state of qubit $B$ has completely the same form with that given in Eq. (11), thus the dependence of MSC on $\left|p_{S}\right|(S=A$ or $B$ ) will also be the same. Of course, the explicit time dependence of the MSC may be different due to the different time dependencies of $p_{S}$ determined by the reservoir spectral density.

Finally, we remark that it is also of great interest to further consider the finite temperature reservoirs. Although it is hard to obtain the two-qubit density operator for this case due to its complexity, one could give a heuristic analysis by considering the Markovian case with $N_{A, B}=1$ for which $\rho_{S}(t)$ can be obtained by solving the master equation in the Lindblad form, and $\rho_{A B}(t)$ can be obtained in a similar way to that of Eq. [10] (see Refs. [65, 66] for more detail). Then for the initial Bell-like states, one can get a MSC behavior qualitatively similar to what we have seen in Sections IV and $\nabla$ The only difference is that the MSC is monotonically decreased with an increase in the reservoir temperature. A general study on the details of the finite temperature effects for the non-Markovian multiple reservoirs is still needed. Besides, it is also worthy to consider the case of two qubits immersed in a common reservoir [37, 44], for which there will be reservoir-mediated interaction between them. The combined and intertwined effects of this indirect interaction and non-Markovianity may induce more rich dynamics of the MSC than that for two independent reservoirs, the details of which will be considered elsewhere.

\section{ACKNOWLEDGMENTS}

This work was supported by the National Natural Science Foundation of China (Grant No. 11675129).
[1] Z. Ficek, S. Swain, Quantum Interference and Coherence: Theory and Experiments, Springer Series in Optical Sciences, Springer, Berlin 2005.

[2] M. A. Nielsen, I. L. Chuang, Quantum Computation and Quantum Information, Cambridge University Press, Cambridge 2010.

[3] A. Streltsov, G. Adesso, M. B. Plenio, Rev. Mod. Phys. 2017, 89, 041003.

[4] M. L. Hu, X. Hu, J. C. Wang, Y. Peng, Y. R. Zhang, H. Fan, Phys. Rep. 2018, 762-764, 1.

[5] T. Baumgratz, M. Cramer, M. B. Plenio, Phys. Rev. Lett. 2014, $113,140401$.

[6] A. Streltsov, U. Singh, H. S. Dhar, M. N. Bera, G. Adesso, Phys. Rev. Lett. 2015, 115, 020403.

[7] X. Yuan, H. Zhou, Z. Cao, X. Ma, Phys. Rev. A 2015, 92, 022124.

[8] C. Napoli, T. R. Bromley, M. Cianciaruso, M. Piani, N. Johnston, G. Adesso, Phys. Rev. Lett. 2016, 116, 150502.

[9] K. Bu, U. Singh, S. M. Fei, A. K. Pati, J. Wu, Phys. Rev. Lett. 2017, 119, 150405.

[10] X. Qi, T. Gao, F. Yan, J. Phys. A 2017, 50, 285301.
[11] K. Bu, N. Anand, U. Singh, Phys. Rev. A 2018, 97, 032342.

[12] A. Streltsov, E. Chitambar, S. Rana, M. N. Bera, A. Winter, M. Lewenstein, Phys. Rev. Lett. 2016, 116, 240405.

[13] J. Ma, B. Yadin, D. Girolami, V. Vedral, M. Gu, Phys. Rev. Lett. 2016, 116, 160407.

[14] C. S. Yu, Phys. Rev. A 2017, 95, 042337.

[15] M. Hillery, Phys. Rev. A 2016, 93, 012111.

[16] K. C. Tan, H. Kwon, C. Y. Park, H. Jeong, Phys. Rev. A 2016, 94, 022329.

[17] Y. Yao, X. Xiao, L. Ge, C. P. Sun, Phys. Rev. A 2015, 92, 022112.

[18] M. L. Hu, H. Fan, Phys. Rev. A 2017, 95, 052106.

[19] X. Hu, A. Milne, B. Zhang, H. Fan, Sci. Rep. 2015, 6, 19365.

[20] X. Hu, H. Fan, Sci. Rep. 2016, 6, 34380.

[21] D. Mondal, T. Pramanik, A. K. Pati, Phys. Rev. A 2017, 95, 010301(R).

[22] M. L. Hu, H. Fan, Phys. Rev. A 2018, 98, 022312.

[23] M. L. Hu, X. M. Wang, H. Fan, Phys. Rev. A 2018, 98, 032317.

[24] E. Chitambar, A. Streltsov, S. Rana, M. N. Bera, G. Adesso, M. Lewenstein, Phys. Rev. Lett. 2016, 116, 070402.

[25] A. Winter, D. Yang, Phys. Rev. Lett. 2016, 116, 120404. 
[26] E. Chitambar, M.-H. Hsieh, Phys. Rev. Lett. 2016, 117, 020402.

[27] B. Regula, K. Fang, X. Wang, G. Adesso, Phys. Rev. Lett. 2018, 121, 010401.

[28] K. Fang, X. Wang, L. Lami, B. Regula, G. Adesso, Phys. Rev. Lett. 2018, 121, 070404.

[29] C. L. Liu, D. L. Zhou, Phys. Rev. A 2020, 101, 012313.

[30] T. Ma, M. J. Zhao, S. M. Fei, G. L. Long, Phys. Rev. A 2016, 94, 042312.

[31] Y. Yao, G. H. Dong, L. Ge, M. Li, C. P. Sun, Phys. Rev. A 2016, 94, 062339.

[32] M. L. Hu, S. Q. Shen, H. Fan, Phys. Rev. A 2017, 96, 052309.

[33] A. Streltsov, H. Kampermann, S. Wölk, M. Gessner, D. Bruß, New J. Phys. 2018, 20, 053058.

[34] S. Kuhr, S. Gleyzes, C. Guerlin, J. Bernu, U. B. Hoff, S. Deléglise, S. Osnaghi, M. Brune, J.-M. Raimondb, Appl. Phys. Lett. 2007, 90, 164101.

[35] S. Maniscalco, F. Francica, R. L. Zaffino, N. L. Gullo, F. Plastina, Phys. Rev. Lett. 2008, 100, 090503.

[36] Z. X. Man, N. B. An, Y. J. Xia, Phys. Rev. A 2014, 90, 062104.

[37] H.-P. Breuer, F. Petruccione, The Theory of Open Quantum Systems, Oxford University Press, Oxford, New York 2002.

[38] H. P. Breuer, E.-M. Laine, J. Piilo, Phys. Rev. Lett. 2009, 103, 210401.

[39] B. Bellomo, R. L. Franco, G. Compagno, Phys. Rev. Lett. 2007, 99, 160502.

[40] B. Bellomo, R. L. Franco, S. Maniscalco, G. Compagno, Phys. Rev. A 2008, 78, 060302.

[41] B. Bellomo, R. L. Franco, G. Compagno, Phys. Rev. A 2008, 78, 062309.

[42] B. Wang, Z. Y. Xu, Z. Q. Chen, M. Feng, Phys. Rev. A 2010, 81, 014101.

[43] F. F. Fanchini, T. Werlang, C. A. Brasil, L. G. E. Arruda, A. O. Caldeira, Phys. Rev. A 2010, 81, 052107.

[44] L. Mazzola, S. Maniscalco, J. Piilo, K.-A. Suominen, B. M. Garraway, Phys. Rev. A 2009, 79, 042302.

[45] M. L. Hu, H. Fan, Phys. Rev. A 2012, 86, 032338.

[46] Y. J. Zhang, W. Han, Y. J. Xia, Y. M. Yu, H. Fan, Sci. Rep. 2015, 5,13359 .

[47] M. L. Hu, H. Fan, Sci. China-Phys. Mech. Astron. 2020, 63,
230322.

[48] M. L. Hu, Y. H. Zhang, H. Fan, Chin. Phys. B 2021, 30, 030308.

[49] A. R. R. Carvalho, J. J. Hope, Phys. Rev. A 2007, 76, 010301.

[50] T. R. Bromley, M. Cianciaruso, G. Adesso, Phys. Rev. Lett. 2015, 114, 210401.

[51] M. L. Hu, H. Fan, Sci. Rep. 2016, 6, 29260.

[52] X. D. Yu, D. J. Zhang, C. L. Liu, D. M. Tong, Phys. Rev. A 2016, 93, 060303(R).

[53] J. Yin, Y. H. Li, S. K. Liao, M. Yang, Y. Cao, L. Zhang, J. G. Ren, W. Q. Cai, W. Y. Liu, S. L. Li, R. Shu, Y. M. Huang, L. Deng, L. Li, Q. Zhang, N. L. Liu, Y. A. Chen, C. Y. Lu, X. B. Wang, F. Xu, J. Y. Wang, C. Z. Peng, A. K. Ekert, J. W. Pan, Nature 2020, 582, 501.

[54] Y. F. Jiao, S. D. Zhang, Y. L. Zhang, A. Miranowicz, L. M. Kuang, H. Jing, Phys. Rev. Lett. 2020, 125, 143605.

[55] I. Bloch, Nature 2008, 453, 1016.

[56] W. Qin, A. Miranowicz, H. Jing, F. Nori, Phys. Rev. Lett. 2021, 127, 093602.

[57] W. C. Wang, Y. L. Zhou, H. L. Zhang, J. Zhang, M. C. Zhang, Y. Xie, C. W. Wu, T. Chen, B. Q. Ou, W. Wu, H. Jing, P. X. Chen, Phys. Rev. A 2021, 103, L020201.

[58] Y. Dai, Y. Dong, Z. Xu, W. You, C. Zhang, O. Gühne, Phys. Rev. Appl. 2020, 13, 054022.

[59] Y. T. Wang, J. S. Tang, Z. Y. Wei, S. Yu, Z. J. Ke, X. Y. Xu, C. F. Li, G. C. Guo, Phys. Rev. Lett. 2017, 118, 020403.

[60] F. Ming, D. Wang, L. J. Li, X. G. Fan, X. K. Song, L. Ye, J. L. Chen, Adv. Quantum Technol. 2021, 4, 2100036.

[61] S. Hill, W. K. Wootters, Phys. Rev. Lett. 1997, 78, 5022.

[62] W. K. Wootters, Phys. Rev. Lett. 1998, 80, 2245.

[63] F. Ming, W. N. Shi, X. G. Fan, L. Ye, D. Wang, J. Phys. A 2021, $54,215302$.

[64] A. J. Leggett, S. Chakravarty, A. T. Dorsey, M. P. A. Fisher, A. Garg, W. Zwerger, Rev. Mod. Phys. 1987, 59, 1.

[65] S. Shresta, C. Anastopoulos, A. Dragulescu, B. L. Hu, Phys. Rev. A 2005, 71, 022109.

[66] B. Bellomo, R. L. Franco, G. Compagno, Phys. Rev. A 2008, 77, 032342. 